\title{
Assessment of DFT Functionals in Predicting Bond Length and Atomization Energy of Catalytically Important Metal Dimers
}

\author{
Selvarengan Paranthaman
}

\author{
Department of Physics and International Research Centre, Kalasalingam University, Krishnankoil 626126, India
} author's e-mail address: psrengan@hotmail.com

RECEIVED: August 2, 2016 * REVISED: December 8, $2016 *$ ACCEPTED: January 24, 2017

Abstract: In the present investigation, the results of extensive benchmarking study of density functional theory (DFT) methods on some catalytically important metal dimers have been reported. The calculations were carried out on $\mathrm{Al}_{2}, \mathrm{Ti}_{2}, \mathrm{~V}_{2}, \mathrm{Cr}_{2}, \mathrm{Mn}_{2}, \mathrm{Fe}_{2}, \mathrm{Co}_{2}, \mathrm{Ni}_{2}, \mathrm{Cu}_{2}$, and $\mathrm{Zn}_{2}$ using DFT functionals such as GGA, meta GGA, hybrid meta GGA along with recently developed Minnesota functionals. The bond length, vibrational frequency, and atomization energy have been calculated for the above studied metal dimers. In order to understand the difference in the performanceof the selected DFT functionals, direct comparison has been made between theoretical and experimental results. Our calculations have shown that, the Minnesota DFT functionals provide better results than other studied functionals. In particular, M06-L functional can be a good choice for the calculations of structural and vibrational frequencies of metal dimers. In the case of atomization energy, MN12-SX show better performance than other studied DFT functionals.

Keywords: transition metals, metal dimers, density functional theory, minnesota functionals, atomization energy.

\section{INTRODUCTION}

$\mathbf{M}$ ETAL clusters have potential applications in the field of catalysis and in molecular electronics. ${ }^{[1-3]}$ In particular, these clusters have been used as heterogeneous catalysts in various industrial applications such as three way catalytic converters, ${ }^{[4]}$ solid oxide fuel cells, ${ }^{[5]}$ and water splitting processes for hydrogen production ${ }^{[6]}$ etc. In general, metal dimers can act as a basic building block of nanosized metal clusters. They can also be treated as smallest model system to study the adsorption on metal surfaces. In the past two decades, extensive theoretical and experimental studies have been performed on these dimers by studying their structural, electronic, optical and magnetic properties. ${ }^{[7-9]}$ However, most of the earlier studies were concentrated on searching low-energy structures and studying their spectroscopic properties using various techniques. ${ }^{[10-15]}$ Experimentally, metal dimers have been generated using molecular beam experiments. Since some metal dimers have less stability, it is difficult to generate those metal dimers in these experiments. Theoretically, metal dimers have been studied using ab initio, and density functional theory (DFT) methods. It is a challenging task to study metal dimers by these theoretical methods. Since these dimers possess near degenerate energy levels, it is difficult to determine the ground state of the system. Earlier studies have shown that, coupled cluster or multireference method provides accurate results for metal dimers. ${ }^{[16,17]}$ Because of the complexity of equations and higher level of excitations, the cost of computation increased while using these methods. Furthermore, it is a difficult task to study nanoscale materials and systems with higher atomic number for ex., ruthenium, gold, etc. using these methods. Hence it is necessary to find out a suitable alternate method with the accuracy of the $\operatorname{CCSD}(T)$ or other multireference methods with less computational time.

In the present study, systematic investigation of metal dimers such as $\mathrm{Al}_{2}, \mathrm{Ti}_{2}, \mathrm{~V}_{2}, \mathrm{Cr}_{2}, \mathrm{Mn}_{2}, \mathrm{Fe}_{2}, \mathrm{Co}_{2}, \mathrm{Ni}_{2}, \mathrm{Cu}_{2}$, and $Z n_{2}$ have been done using DFT methods. The main objective is to find out suitable DFT functional for the studies on metallic systems. DFT is an appropriate method to study the metallic systems. These methods describe exchange- 
correlation well, which is necessary to study metallic systems. Earlier, few benchmarking studies were performed to assess the performance of DFT functionals as well as basis set on transition metal dimers. For instance, Du et al. have studied the performance difference of various DFT functionals for transition metal dimers. ${ }^{[18]}$ Similarly, other DFT studies were also performed on metal dimers. ${ }^{[17,19-21]}$ To the best of my knowledge, till now no systematic DFT assessment study has been done on catalytically important metal dimers. In particular, recently developed Minnesota DFT functionals and other recently developed hybrid meta GGA functionals have not been assessed. Hence, in the present study, various DFT functionals has been assessed to study the structure and energetical properties of $\mathrm{Al}_{2}, \mathrm{Ti}_{2}, \mathrm{~V}_{2}$, $\mathrm{Cr}_{2}, \mathrm{Mn}_{2}, \mathrm{Fe}_{2}, \mathrm{Co}_{2}, \mathrm{Ni}_{2}, \mathrm{Cu}_{2}$, and $\mathrm{Zn}_{2}$ metal dimers. These metal dimers are catalytically important metal systems. The clusters of these metals have been widely investigated for their applications in catalysis. Moreover, the greater advent of DFT functionals and the existence of accurate experimental data have led me to perform this assessment study on metal dimers.

\section{Computational Details}

The geometry of the studied metal dimers were optimized using the DFT functionals such as BLYP, ${ }^{22,23]} \mathrm{BP} 86,{ }^{222,24]}$ BPW91, ${ }^{[22,25]}$ B97D, ${ }^{[26]}$ PBE, ${ }^{[27,28] ~ m P W P W 91, ~}{ }^{[25,29]}$ TPSS, ${ }^{[30]}$ N12, ${ }^{[31]}$ M06-L,, ${ }^{[32] ~ M N 12-L,[33] ~ B H a n d H, ~}{ }^{[22,23]}$ B3LYP, ${ }^{[23,34]}$ B3P86, [24,34] B3PW91,[34,35] PBE0,[36] mPW1PW91, [25,29] M05, ${ }^{[37]} \mathrm{M} 05-2 X_{,}{ }^{[38]} \mathrm{M} 06,{ }^{[39]} \mathrm{M} 06-2 X_{,}{ }^{[39]}$ TPSSh, ${ }^{[40]} \mathrm{MN} 12-$ $S X .{ }^{[41]}$ In order to get accurate results for metallic systems, researchers mostly use high level basis set in their calculations. However, it is quite impossible to study larger sized metallic systems with higher level basis set such as correlation consistent or triple zeta valence polarization functions. It is necessary to find out an alternate combination of DFT functional with low or medium level basis set to study the metal systems. Hence, in the present study all electron 6$31 \mathrm{G}(\mathrm{d})$ basis set has been used for the calculations. The spin restricted and unrestricted formalisms were used to calculate the singlet and higher spin states, respectively. The initial geometries of the studied metal dimers were taken from the literature. ${ }^{[18,20]}$ Vibrational frequency calculations were performed for all metal dimers to identify the minimum energy structure. The atomization energy (AE) of the metal dimers was also calculated.

$$
\begin{aligned}
& \mathrm{AE}=\mathrm{E}\left(\mathrm{X}_{n}\right)-\mathrm{nE}(\mathrm{X}) \\
& (n=2, X=\mathrm{Al}, \mathrm{Ti}, \mathrm{V}, \mathrm{Cr}, \mathrm{Mn}, \mathrm{Fe}, \mathrm{Co}, \mathrm{Ni}, \mathrm{Cu}, \text { and } \mathrm{Zn} \text { ) }
\end{aligned}
$$

The atomization energy is also termed as dissociation energy in the literature. All calculations were performed using the Gaussian09 program. ${ }^{[42]}$

\section{RESULTS AND DISCUSSION}

The metal dimers $\mathrm{Al}_{2}, \mathrm{Ti}_{2}, \mathrm{~V}_{2}, \mathrm{Cr}_{2}, \mathrm{Mn}_{2}, \mathrm{Fe}_{2}, \mathrm{Co}_{2}, \mathrm{Ni}_{2}, \mathrm{Cu}_{2}$, and $\mathrm{Zn}_{2}$ were optimized using the DFT functionals. The selected DFT functionals are Generalized Gradient Approximation (GGA) (BLYP, BP86, BPW91, B97D, PBE, mPWPW91), hybrid GGA (B3LYP, B3P86, B3PW91, PBE0, mPW1PW91, BHandH), meta-GGA (TPSS, M06-L), hybrid-meta GGA (M05, M05-2X, M06, M06-2X, TPSSh), Non separable Gradient Approximation (NGA) (N12), meta NGA (MN12-L), and Range-separated hybrid meta NGA functionals (MN12$S X)$. The calculated bond length, vibrational frequency, and atomization energy along with available experimental results are given in Table 1, 2, and 3 respectively. The overall mean absolute deviation (MAD) error, i.e. difference between experimental and theoretical results has been calculated. The MAD error of bond length, vibrational frequency and atomization energy of the studied metal dimers is shown in Figure 1. The recommended DFT functionals for the calculation of bond length, vibrational frequency and atomization energy of the studied metal dimers are summarized in Table 4.

\section{$\mathrm{Al}_{2}$ Dimer}

$\mathrm{Al}_{2}$ dimer is a well-studied system; it can be noted from the literature. The reason behind this is, Al clusters show superatom behavior. ${ }^{[43]}$ Recently, Selvarengan et al. ${ }^{[21]}$ have performed DFT assessment study on $\mathrm{Al}_{2}-\mathrm{Al}_{10}$ clusters in both neutral and anion form. Apart from this, there are other experimental and theoretical studies reported in the literature. ${ }^{[44,45]}$ The main focus of my study is, to understand the difference in the performance of recently developed DFT functionals. Therefore, it is necessary to compare the calculated results with the available experimental results. My study has shown that, the bond length predicted by hybrid $\mathrm{BHandH}$ functional is in close agreement with experimental results. However, the earlier study on $\mathrm{Al}_{2}$ dimer ${ }^{[21]}$ has shown that better performed $\omega B 97 X D$ functional with higher level def2-TZVPP basis set slightly overestimated the $\mathrm{Al}-\mathrm{Al}$ bond length. This indicates that, even using the lower level basis set, one can get accurate theoretical results. Furthermore, vibrational frequency predicted by Minnesota functional M05-2X $\left(285.88 \mathrm{~cm}^{-1}\right)$ coincides well with the experimental value $\left(284.20 \mathrm{~cm}^{-1}\right)$. In this case, BHandH functional slightly overestimates the vibrational frequency $\left(286.39 \mathrm{~cm}^{-1}\right)$. The atomization energy predicted by GGA BLYP functional coincides very well with the experimental value. On the other hand, the atomization energy predicted by $\mathrm{BHandH}$ and $\mathrm{M} 05-2 \mathrm{X}$ functionals is found to be slightly higher. In summary, BHandH and M05-2X functionals shows best performance while studying the structure and vibrational frequency of $\mathrm{Al}_{2}$ dimer. 


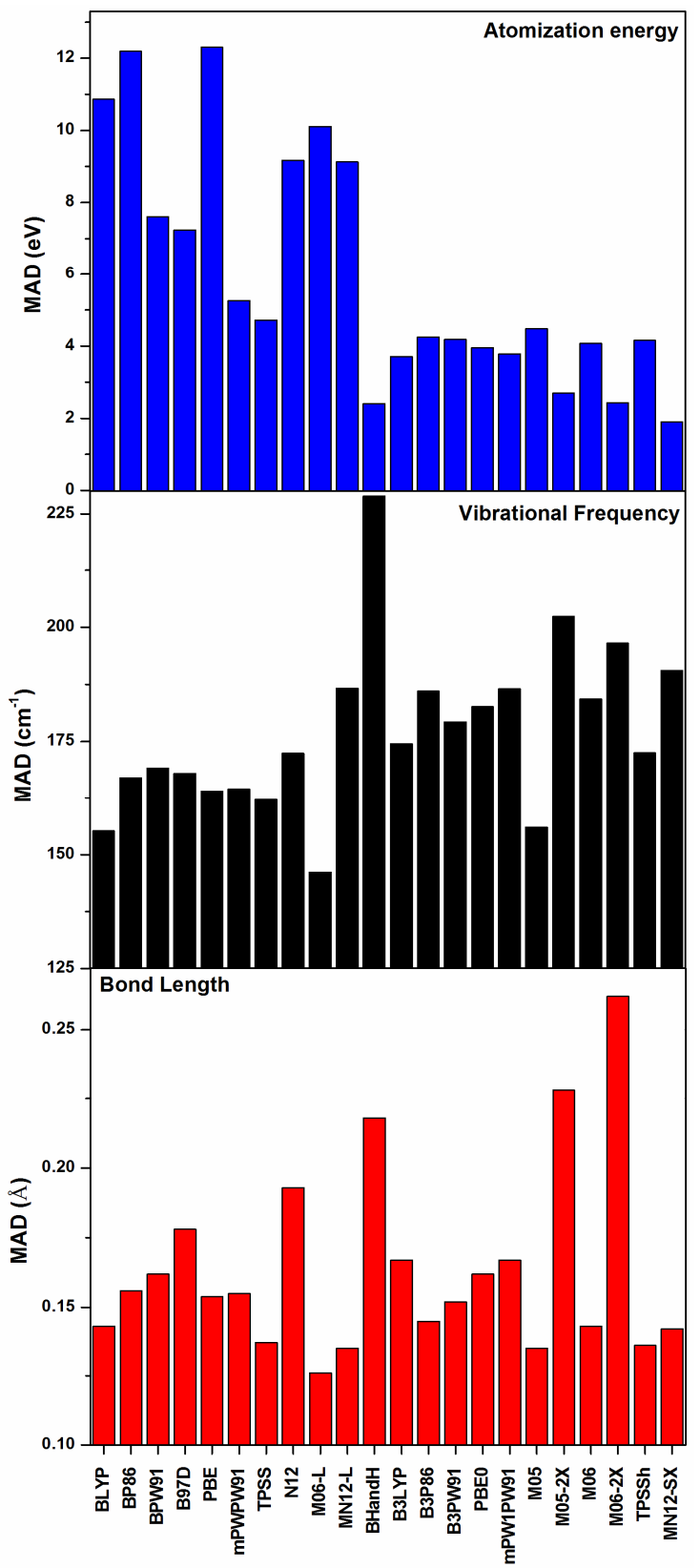

Figure 1. Mean absolute deviation (MAD) of bond lengths in $\AA$, vibrational frequency in $\mathrm{cm}^{-1}$ and Atomization energy in eV of studied metal dimers.

\section{$\mathrm{Ti}_{2}$ Dimer}

Present calculations have shown that, the Ti-Ti bond length of $\mathrm{Ti}_{2}$ dimer predicted by Minnesota M06-L functional is in close agreement with the experimental value. ${ }^{[46]}$ The difference in the Ti-Ti bond length between calculated and experimental results is $0.04 \AA$. The other DFT functionals that are taken for this study are found to underestimate the TiTi bond length. The results obtained from this work are in agreement with previous theoretical studies. Earlier, Gutsev and Bauschlicher have assessed DFT functionals for homo nuclear $3 \mathrm{~d}$ metal dimers. ${ }^{[20]}$ They selected few GGA functionals with $6-311+G(d)$ and Triple- $\zeta$ correlation consistent basis sets for their study and these selected DFT functionals underestimate the Ti-Ti bond length. They have obtained $\mathrm{Ti}-\mathrm{Ti}$ bond length to be $1.920 \AA$ using BLYP/6$311+G(d)$ basis set. In my study BLYP functional with 6-31G(d) basis set predicts Ti-Ti bond length to be $1.877 \AA$. This indicates that the basis set plays an important role in determining the bond length of $\mathrm{Ti}_{2}$ dimer. The most accurate theoretical result was predicted by Hubner et al.[47] They have used the CASSCF method with ANO basis set for their calculations. In the case of vibrational frequency, except M06-L functional, all the studied DFT functionals overestimates vibrational frequency. The vibrational frequency calculated by M06-L is in close agreement with experimental data ${ }^{[48]}$ and also coincides well with the previous DFT study. ${ }^{[20]}$ The atomization energy, calculated by M06-2X and M05-2X is comparable with the experimental data. ${ }^{[49]}$ All the other studied functionals overestimate the atomization energy. This indicates that, these functionals with the lower basis set have difficulty in estimating the atomization energy. Hence, based on all these results, it could be concluded that Minnesota functionals M06-L, M06-2X, and M05-2X show better performance for studying structural and energy parameters of $\mathrm{Ti}_{2}$ dimer rather than any other studied DFT functionals. However, it could be much better if one may use highly correlated ab initio methods to get accurate results of $\mathrm{Ti}_{2}$ dimer and $\mathrm{Ti}$ containing systems.

\section{$\mathbf{V}_{2}$ Dimer}

In the case of $V_{2}$ dimer, the present calculations have shown that the $\mathrm{V}-\mathrm{V}$ bond length predicted by TPSS functional coincides well with experimenta|[50] and CASSCF results. ${ }^{[51]}$ All other studied DFT functionals underestimate the $\mathrm{V}-\mathrm{V}$ bond length in $\mathrm{V}_{2}$ dimer. Earlier Barden et al. found that the bond length calculated from their DFT study coincides well with experimental value.[16] In particular, the $\mathrm{V}-\mathrm{V}$ bond length calculated by BP86 functional coincides very well with experimental value. They have used basis sets constructed from Wachters sets. Similarly, Gutsev and Bauschlicher have predicted the $\mathrm{V}-\mathrm{V}$ bond length of $1.76 \AA$ in $V_{2}$ dimer. ${ }^{[20]}$ They have used BLYP/6-311+G(d) level of theory for their calculations. In their study, BLYP functional shows good performance for bond length, and overestimates vibrational frequency and atomization energy. In the case of vibrational frequency, the DFT functionals that are taken for study are found to overestimate the vibrational frequency. This is quite understandable, because the DFT assessment study done earlier have also mentioned that, these functionals overestimate the vibrational frequency of $\mathrm{V}_{2}$ dimer. However, vibrational frequency calculated by 
Table 1. Optimized bond lengths (in $\AA$ ) of studied metal dimers

\begin{tabular}{|c|c|c|c|c|c|c|c|c|c|c|c|c|}
\hline Functionals & ${ }^{3} \mathrm{Al}_{2}$ & ${ }^{3} \mathrm{Ti}_{2}$ & ${ }^{3} V_{2}$ & ${ }^{1} \mathrm{Cr}_{2}$ & ${ }^{11} \mathrm{Mn}_{2}$ & ${ }^{7} \mathrm{Fe}_{2}$ & ${ }^{9} \mathrm{Fe}_{2}$ & ${ }^{5} \mathrm{CO}_{2}$ & ${ }^{7} \mathrm{CO}_{2}$ & ${ }^{3} \mathrm{Ni}_{2}$ & ${ }^{1} \mathrm{Cu}_{2}$ & ${ }^{1} \mathrm{Zn}_{2}$ \\
\hline BLYP & 2.801 & 1.877 & 1.660 & 1.562 & 3.260 & 1.884 & 2.027 & 1.979 & 1.917 & 2.026 & 2.017 & 2.573 \\
\hline BP86 & 2.773 & 1.857 & 1.694 & 1.550 & 3.148 & 1.869 & 2.010 & 1.900 & 1.902 & 2.011 & 2.006 & 2.519 \\
\hline BPW91 & 2.766 & 1.857 & 1.639 & 1.551 & 3.182 & 1.874 & 2.014 & 1.904 & 1.906 & 1.983 & 2.010 & 2.550 \\
\hline B97D & 2.800 & 1.855 & 1.678 & 1.537 & 3.346 & 2.010 & 2.014 & 1.907 & 1.973 & 1.947 & 2.025 & 2.844 \\
\hline PBE & 2.763 & 1.859 & 1.693 & 1.550 & 3.171 & 1.873 & 2.013 & 1.904 & 1.905 & 2.014 & 2.009 & 2.546 \\
\hline mPWPW91 & 2.764 & 1.856 & 1.694 & 1.551 & 3.163 & 1.873 & 2.013 & 1.903 & 1.904 & 2.014 & 2.009 & 2.541 \\
\hline TPSS & 2.745 & 1.863 & 1.697 & 1.550 & 3.197 & 1.870 & 2.006 & 1.960 & 1.896 & 2.054 & 2.002 & 2.513 \\
\hline N12 & 2.722 & 1.796 & 1.668 & 1.532 & 3.066 & 1.942 & 2.113 & 1.953 & 1.963 & 1.928 & 2.001 & 2.636 \\
\hline M06-L & 2.715 & 1.903 & 1.692 & 1.540 & 3.262 & 2.009 & 2.044 & 1.968 & 1.988 & 2.070 & 2.016 & 2.551 \\
\hline MN12-L & 2.772 & 1.852 & 1.663 & 1.517 & 3.234 & 1.850 & 1.983 & 1.983 & 1.927 & 2.036 & 1.994 & 2.394 \\
\hline BHandH & 2.706 & 1.787 & 1.578 & 1.476 & 3.617 & 1.948 & 1.945 & 1.816 & 1.783 & 1.973 & 2.014 & 2.800 \\
\hline B3LYP & 2.764 & 1.842 & 1.671 & 1.527 & 3.623 & 1.991 & 1.995 & 1.951 & 1.863 & 2.085 & 2.024 & 2.731 \\
\hline B3Р86 & 2.736 & 1.824 & 1.660 & 1.517 & 3.403 & 1.975 & 1.980 & 1.938 & 1.850 & 2.070 & 2.014 & 2.666 \\
\hline B3PW91 & 2.741 & 1.830 & 1.663 & 1.519 & 3.469 & 1.982 & 1.987 & 1.944 & 1.856 & 2.078 & 2.020 & 2.708 \\
\hline PBEO & 2.733 & 1.826 & 1.655 & 1.511 & 3.500 & 1.979 & 1.982 & 1.939 & 1.846 & 2.083 & 2.023 & 2.759 \\
\hline mPW1PW91 & 2.733 & 1.824 & 1.607 & 1.512 & 3.508 & 1.978 & 1.982 & 1.939 & 1.845 & 2.084 & 2.024 & 2.752 \\
\hline M05 & 2.737 & 1.871 & 1.664 & 1.516 & 3.453 & 2.006 & 2.007 & 1.977 & 1.871 & 2.175 & 2.039 & 2.724 \\
\hline M05-2X & 2.740 & 1.809 & 1.626 & 1.485 & 3.777 & 1.972 & 1.967 & 1.860 & 1.803 & 2.208 & 2.064 & 3.030 \\
\hline M06 & 2.729 & 1.852 & 1.660 & 1.517 & 3.313 & 1.993 & 2.003 & 1.946 & 1.863 & 1.996 & 2.023 & 2.561 \\
\hline M06-2X & 2.730 & 1.804 & 1.625 & 1.486 & 3.978 & 1.988 & 1.984 & 1.876 & 1.819 & 2.240 & 2.076 & 3.184 \\
\hline TPSSh & 2.734 & 1.849 & 1.680 & 1.534 & 3.337 & 1.987 & 1.994 & 1.949 & 1.872 & 2.063 & 2.008 & 2.600 \\
\hline MN12-SX & 2.759 & 1.847 & 1.657 & 1.510 & 3.329 & 1.966 & 1.973 & 1.995 & 1.836 & 2.064 & 2.015 & 2.604 \\
\hline Exp. & $2.701^{[44]}$ & $\begin{array}{l}1.943 \pm \\
0.001^{[46]}\end{array}$ & $1.770^{[50]}$ & $1.679[52]$ & $\leq 3.4^{[57]}$ & $\begin{array}{l}2.020 \pm \\
0.020^{[59]}\end{array}$ & - & $2.310^{[62]}$ & - & $\begin{array}{l}2.155 \pm \\
0.001^{[14]}\end{array}$ & $2.219[67]$ & $\begin{array}{l}2.350 \pm \\
0.080^{[69]}\end{array}$ \\
\hline
\end{tabular}

highly correlated CASSCF method yields comparable results. This shows that, one need to use higher basis set with DFT functional or highly correlated ab initio method to get the accurate vibrational frequency for this dimer. Also, the atomization energy, calculated by Minnesota functionals M05-2X and M06-2X performs better rather than the other studied DFT functionals. Apart from these two functionals all the other functionals that are taken for my study are found to overestimate the atomization energy. Earlier Gutsev and Bauschlicher have also obtained high atomization energy for all the functionals except BPW91 functional.[20] In their study, the atomization energy, calculated by BPW91 functional is in agreement with the experimental data.

\section{$\mathrm{Cr}_{2}$ Dimer}

Since $\mathrm{Cr}_{2}$ dimer possesses sectuple bond ${ }^{[17]}$ and has a shorter bond length than the other metal dimers that are taken for this study. The present calculations have shown that, all the functionals that are taken in this study underestimate the $\mathrm{Cr}-\mathrm{Cr}$ bond length. For instance, BLYP functional gives $1.562 \AA$, which is very much lower than the experimental value $1.679 \AA$. . ${ }^{[52]}$ Earlier, Gutsev and Bauschlicher have used BLYP functional with $6-311+G(d)$ basis set and obtained the value $1.710 \AA$ for the $\mathrm{Cr}-\mathrm{Cr}$ bond length. ${ }^{[20]}$ In the case of vibrational frequency of $\mathrm{Cr}_{2}$ dimer, no functional predicts accurate result. Earlier Casey et al. have obtained experimental vibrational frequency for $\mathrm{Cr}_{2}$ dimer. ${ }^{[53]}$ All the functionals that are taken for my study are found to overestimate the vibrational frequency. Also, a significant deviation is noted in the vibrational frequency values calculated from this study as well as Gutsev and Bauschlicher study. Eventhough, their results are comparable with experimental results, DFT functionals used in their study underestimates the vibrational frequency of $\mathrm{Cr}_{2}$ dimer. Similarly, DFT functionals used in this study overestimates the atomization energy. The experimental atomization energy was calculated by Hilpert et al. ${ }^{[54]}$ Earlier Mills et al. have shown that DFT methods fail to predict binding energy in the metallic systems. ${ }^{[55]}$ This could be because these methods were unable to describe the charge transfer between the metal and other atoms. ${ }^{[56]}$ Further, it may also be noted that the error in the atom energy calculation leads to an error in the atomization energy values. This error arises may be because, the transition metals have a large number of low lying electronic states. In that case, one needs to use relativistic corrections to get the accurate results. The relativistic error may increase as the size of the atom increases. Due to these discrepancies, large structural and energetical values for $\mathrm{Cr}_{2}$ dimer have been obtained. In 
Table 2. Calculated vibrational frequencies (in $\mathrm{cm}^{-1}$ ) of studied metal dimers

\begin{tabular}{|c|c|c|c|c|c|c|c|c|c|c|c|c|}
\hline Functionals & ${ }^{3} \mathrm{Al}_{2}$ & ${ }^{3} \mathrm{Ti}_{2}$ & ${ }^{3} V_{2}$ & ${ }^{1} \mathrm{Cr}_{2}$ & ${ }^{11} \mathrm{Mn}_{2}$ & ${ }^{7} \mathrm{Fe}_{2}$ & ${ }^{9} \mathrm{Fe}_{2}$ & ${ }^{5} \mathrm{CO}_{2}$ & ${ }^{7} \mathrm{CO}_{2}$ & ${ }^{3} \mathrm{Ni}_{2}$ & ${ }^{1} \mathrm{Cu}_{2}$ & ${ }^{1} \mathrm{Zn}_{2}$ \\
\hline BLYP & 241.19 & 529.36 & 746.43 & 923.03 & 76.56 & 499.78 & 421.32 & 425.02 & 446.43 & 407.70 & 410.21 & 118.93 \\
\hline BP86 & 255.61 & 545.59 & 750.51 & 946.71 & 86.41 & 510.54 & 435.94 & 465.68 & 459.77 & 417.73 & 418.54 & 122.15 \\
\hline BPW91 & 259.01 & 541.22 & 794.13 & 943.36 & 83.97 & 505.37 & 432.44 & 461.55 & 456.35 & 418.92 & 415.58 & 118.32 \\
\hline B97D & 235.92 & 554.76 & 757.58 & 969.29 & 101.07 & 428.94 & 421.07 & 445.39 & 410.37 & 442.78 & 394.87 & 53.72 \\
\hline PBE & 260.49 & 539.22 & 747.78 & 945.33 & 85.30 & 506.03 & 433.49 & 462.86 & 457.28 & 416.75 & 416.58 & 119.36 \\
\hline mPWPW91 & 259.57 & 543.39 & 748.73 & 944.03 & 85.45 & 506.88 & 433.56 & 462.86 & 457.43 & 415.83 & 416.86 & 119.08 \\
\hline TPSS & 265.49 & 543.09 & 746.46 & 948.19 & 81.83 & 507.72 & 442.79 & 442.85 & 468.88 & 405.97 & 421.41 & 118.36 \\
\hline N12 & 270.95 & 642.61 & 786.75 & 952.53 & 110.83 & 413.67 & 353.17 & 435.92 & 418.74 & 466.61 & 404.75 & 124.62 \\
\hline M06-L & 267.21 & 498.35 & 748.39 & 949.00 & 78.60 & 431.34 & 405.37 & 425.52 & 403.71 & 386.32 & 404.89 & 108.81 \\
\hline MN12-L & 250.70 & 561.36 & 814.96 & 1036.66 & 88.33 & 484.39 & 452.79 & 399.67 & 461.54 & 419.63 & 421.54 & 167.74 \\
\hline BHandH & 286.39 & 671.92 & 944.09 & 1178.84 & 52.96 & 483.89 & 497.75 & 494.34 & 578.47 & 416.17 & 401.50 & 78.37 \\
\hline B3LYP & 259.10 & 583.90 & 805.34 & 1020.03 & 47.91 & 453.32 & 451.32 & 447.71 & 495.78 & 376.81 & 402.36 & 86.41 \\
\hline B3P86 & 271.88 & 601.68 & 822.42 & 1041.54 & 66.92 & 467.50 & 465.64 & 457.71 & 506.72 & 386.52 & 409.16 & 105.33 \\
\hline B3РW91 & 270.88 & 589.95 & 814.20 & 1035.33 & 57.81 & 460.74 & 459.15 & 451.83 & 502.24 & 380.49 & 404.43 & 93.41 \\
\hline PBEO & 275.91 & 597.33 & 829.00 & 1058.08 & 57.38 & 463.80 & 464.85 & 454.05 & 512.73 & 375.96 & 401.55 & 82.50 \\
\hline mPW1PW91 & 275.29 & 598.01 & 867.04 & 1056.33 & 56.11 & 464.12 & 464.76 & 453.23 & 513.20 & 374.87 & 401.04 & 82.99 \\
\hline M05 & 262.84 & 514.87 & 781.62 & 1007.04 & 65.05 & 441.52 & 441.17 & 438.29 & 503.62 & 328.30 & 400.30 & 99.87 \\
\hline M05-2X & 285.88 & 669.27 & 907.15 & 1165.63 & 49.74 & 470.10 & 479.63 & 449.20 & 553.41 & 301.95 & 379.68 & 64.42 \\
\hline M06 & 262.43 & 545.49 & 803.95 & 1032.08 & 82.03 & 461.78 & 447.45 & 464.41 & 495.15 & 433.46 & 413.30 & 124.72 \\
\hline M06-2X & 279.42 & 657.50 & 907.34 & 1155.69 & 40.98 & 451.15 & 465.66 & 447.67 & 534.81 & 284.18 & 371.31 & 57.54 \\
\hline TPSSh & 271.50 & 566.37 & 779.03 & 993.75 & 76.80 & 459.06 & 454.81 & 450.23 & 489.96 & 394.22 & 415.33 & 114.54 \\
\hline MN12-SX & 249.03 & 586.55 & 843.17 & 1079.40 & 86.40 & 482.82 & 471.02 & 407.55 & 516.69 & 402.24 & 407.69 & 110.01 \\
\hline Exp. & $284.20^{[44]}$ & $407.90^{[48]}$ & $537.50^{[48]}$ & $\begin{array}{r}480.60 \pm \\
0.50[53]\end{array}$ & $68.10^{[58]}$ & $299.70^{[60]}$ & - & $\begin{array}{r}296.80 \pm \\
0.50^{[63]}\end{array}$ & - & $\begin{array}{c}259.2 \pm \\
3^{[65]}\end{array}$ & $266.46^{[67]}$ & $80 \pm 1^{[70]}$ \\
\hline
\end{tabular}

summary, the lower level basis set is not suitable for the calculation of structural and energetical parameters in this dimer. From the earlier studies, it is clear that even using higher level $a b$ initio calculations also, one could not produce accurate results in this dimer. In order to get the accurate results, one can use larger basis sets combined with recently developed DFT functionals.

\section{$\mathrm{Mn}_{2}$ dimer}

In the case of $\mathrm{Mn}_{2}$ dimer, it is noted that most of the studied DFT functionals predict the Mn-Mn bond length accurately. For instance, the $\mathrm{Mn}-\mathrm{Mn}$ bond length calculated by hybrid GGA B3P86 functional coincides very well with the experimental value. The experimental bond length of $\mathrm{Mn}_{2}$ dimer has been obtained using electron spin resonance spectroscopy technique by Cheeseman et al. ${ }^{[57]}$ In the case of vibrational frequency, B3P86 functional predicts 66.92 $\mathrm{cm}^{-1}$, which is in good agreement with Kirkwood et al. experimental data. ${ }^{[58]}$ This study indicates that hybrid GGA B3P86 functional accurately predicts both the bond length and vibrational frequency of $\mathrm{Mn}_{2}$ dimer. While considering the atomization energy, earlier experimental studies produced different results. For instance, Morse has obtained the atomization energy to be $0.80 \mathrm{eV}$, and Kirkwood et al. has predicted it to be $0.1 \mathrm{eV}$. In the present study, all the
DFT functionals taken for the study overestimate the atomization energy. The calculated values are very high when compared with the available experimental values. This indicates that, studied DFT functionals combined with 6$31 \mathrm{G}(\mathrm{d})$ basis set is not suitable for the calculations of energy parameters. Hence it is recommended that, the higher-level basis sets either correlation consistent or Triple zeta valence polarization functions could be used to get the accurate atomization energy in this metal system.

\section{$\mathrm{Fe}_{2}$ dimer}

Du et al. have shown that, there are two electronic spin states 7 and 9 in $\mathrm{Fe}_{2}$ dimer. ${ }^{[18]}$ While studying the stability of those spin states, the selected DFT functionals produce mixed results. Our calculations have shown that, except Minnesota MN12-L functional all other functionals taken for this study predict spin 9 state to be more stable than spin 7 state. The MN12-L functional shows, spin 7 state is more stable than spin 9 state. However, the energy difference between these two states is small. The calculated results show that, GGA BPW91 and B97D functionals predict the bond length, comparable with the available experimental value. ${ }^{[59]}$ Alternatively, their hybrid version underestimates the $\mathrm{Fe}-\mathrm{Fe}$ bond length of $\mathrm{Fe}_{2}$ dimer. In the case of vibrational frequency, experimental vibrational frequency of 
Table 3. Calculated Atomization energy (in eV) of studied metal dimers

\begin{tabular}{|c|c|c|c|c|c|c|c|c|c|c|}
\hline Functionals & $\mathrm{Al}_{2}$ & $\mathrm{Ti}_{2}$ & $V_{2}$ & $\mathrm{Cr}_{2}$ & $\mathrm{Mn}_{2}$ & $\mathrm{Fe}_{2}$ & $\mathrm{CO}_{2}$ & $\mathrm{Ni}_{2}$ & $\mathrm{Cu}_{2}$ & $\mathrm{Zn}_{2}$ \\
\hline BLYP & 1.36 & 5.97 & 6.89 & 74.12 & 10.04 & 9.02 & 4.45 & 7.87 & 3.46 & 0.33 \\
\hline BP86 & 1.54 & 6.67 & 8.76 & 74.57 & 10.35 & 9.45 & 12.76 & 8.42 & 3.85 & 0.43 \\
\hline BPW91 & 1.53 & 6.69 & 7.63 & 12.88 & 27.39 & 9.49 & 12.44 & 8.70 & 3.85 & 0.39 \\
\hline B97D & 1.48 & 6.59 & 8.76 & 13.21 & 28.39 & 10.51 & 4.97 & 6.56 & 6.55 & 0.28 \\
\hline PBE & 1.61 & 6.75 & 8.88 & 74.51 & 10.77 & 9.69 & 12.88 & 8.53 & 3.88 & 0.45 \\
\hline mPWPW91 & 1.57 & 6.74 & 8.76 & 12.95 & 10.56 & 9.53 & 4.50 & 8.47 & 3.90 & 0.43 \\
\hline TPSS & 1.51 & 6.39 & 8.06 & 9.75 & 10.16 & 8.75 & 4.41 & 8.84 & 3.72 & 0.45 \\
\hline N12 & 1.64 & 20.73 & 22.93 & 13.31 & 25.03 & 6.48 & 4.80 & 6.68 & 4.68 & 0.27 \\
\hline M06-L & 1.58 & 2.43 & 4.85 & 73.10 & 8.69 & 8.94 & 4.08 & 8.55 & 3.19 & 0.50 \\
\hline MN12-L & 1.87 & 3.60 & 4.72 & 69.03 & 6.64 & 4.57 & 3.46 & 7.70 & 3.92 & 0.61 \\
\hline BHandH & 1.47 & 3.07 & 2.19 & 4.82 & 12.41 & 6.81 & 1.66 & 1.91 & 1.24 & 0.21 \\
\hline B3LYP & 1.32 & 4.47 & 5.82 & 8.35 & 10.58 & 7.31 & 4.43 & 7.18 & 2.24 & 0.18 \\
\hline B3Р86 & 1.49 & 5.21 & 6.67 & 9.41 & 11.14 & 8.09 & 4.70 & 7.69 & 2.70 & 0.27 \\
\hline B3PW91 & 1.46 & 5.05 & 6.46 & 9.17 & 11.39 & 8.13 & 4.61 & 7.64 & 2.58 & 0.23 \\
\hline PBEO & 1.50 & 4.72 & 5.79 & 8.40 & 11.74 & 7.97 & 4.40 & 7.44 & 2.25 & 0.23 \\
\hline mPW1PW91 & 1.47 & 4.70 & 4.60 & 8.28 & 11.58 & 7.81 & 4.29 & 7.43 & 2.28 & 0.22 \\
\hline M05 & 1.48 & 4.63 & 6.66 & 9.28 & 13.09 & 9.74 & 5.29 & 6.14 & 3.13 & 0.22 \\
\hline M05-2X & 1.46 & 2.19 & 2.67 & 3.94 & 14.63 & 6.74 & 0.10 & 3.47 & 0.80 & 0.11 \\
\hline M06 & 1.48 & 4.42 & 6.50 & 8.72 & 12.62 & 8.87 & 4.68 & 5.10 & 2.72 & 0.44 \\
\hline M06-2X & 1.45 & 0.92 & 2.52 & 3.37 & 13.88 & 5.89 & 0.70 & 2.97 & 0.33 & 0.10 \\
\hline TPSSh & 1.47 & 5.60 & 6.90 & 7.95 & 10.73 & 8.21 & 5.14 & 7.08 & 3.04 & 0.34 \\
\hline MN12-SX & 1.68 & 2.74 & 3.69 & 5.19 & 6.73 & 3.45 & 1.74 & 5.96 & 2.36 & 0.30 \\
\hline Exp. & $1.36^{[72,44]}$ & $\begin{array}{l}1.54 \\
\pm 0.18^{[49]}\end{array}$ & $\begin{aligned} & 2.753 \\
\pm & 0.001^{[50]}\end{aligned}$ & $\begin{array}{l}1.44 \\
\pm 0.05^{[54]}\end{array}$ & $\leq 0.80^{[7]}$ & $\begin{array}{l}1.15 \\
\pm 0.09^{[61]}\end{array}$ & $\begin{array}{l}1.69 \\
\pm 0.26^{[62]}\end{array}$ & $\begin{aligned} & 2.068 \\
\pm & 0.01^{[66]}\end{aligned}$ & $\begin{array}{c}2.01 \\
\pm 0.08^{[7]}\end{array}$ & $0.056^{[71]}$ \\
\hline
\end{tabular}

$\mathrm{Fe}_{2}$ dimer is calculated by Moskowits et al. ${ }^{[60]}$ The results produced by NGA N12 functional are consistent with the available experimental data. All the DFT functionals taken in this study yield large atomization energy and does not produce accurate experimental result. Experimentally, Lian et al. have obtained atomization energy. ${ }^{[61]}$ In summary, GGA and NGA functionals accurately predict the bond length and vibrational frequency values. No DFT functional with $6-31 \mathrm{G}(\mathrm{d})$ basis set is suitable for the calculation of atomization energy. Hence it is recommended that, one may use higher level basis sets to get the accurate energy values.

\section{$\mathrm{CO}_{2}$ dimer}

In the case of $\mathrm{CO}_{2}$ dimer, earlier studies have shown that two electronic states 5 and 7 exist in $\mathrm{CO}_{2}$ dimer. In this study, while studying the structural stability of this dimer, mixed results have been obtained. Earlier theoretical studies concluded that spin 5 state is the ground state. Most the GGA functionals taken in this study predict spin 7 state to be stable, whereas the hybrid GGA functionals show spin 5 to be energetically more stable. In accordance with the earlier theoretical studies, Spin 5 state has been considered for atomization energy calculations. The optimized bond length and vibrational frequency of two electronic states in
$\mathrm{Co}_{2}$ dimer are given in Table 1 and 2 along with experimental results. ${ }^{[62,63]}$ Theoretically, Shim and Gingerich have calculated $\mathrm{Co}-\mathrm{Co}$ bond length of $\mathrm{Co}_{2}$ dimer using high level singles, doubles configuration interaction (SDCl) method. ${ }^{[64]}$ While considering the difference in the performance of the studied DFT functionals, all the functionals underestimate the bond length and overestimate the vibrational frequency. The difference between theoretical and experimental results is $\sim 0.3 \AA$, and $\sim 100 \mathrm{~cm}^{-1}$ for bond length and vibrational frequency, respectively. In the case of atomization energy, hybrid BHandH and Minnesota MN12-SX functionals give better results than other studied DFT functionals. Since $\mathrm{Co}_{2}$ dimer possesses high electronic spin state, calculations could be performed using higher level basis set or highly correlated ab initio method for this dimer.

\section{$\mathrm{Ni}_{2}$ dimer}

In this case, most of the studied functionals produce consistent results. For instance, $\mathrm{Ni}-\mathrm{Ni}$ bond length of $\mathrm{Ni}_{2}$ dimer calculated by Minnesota M05 functional gives better result rather than the other studied functionals. In the case of vibrational frequency, all the studied functionals overestimate the vibrational frequency except M06-2X functional. The vibrational frequency calculated by highly parametrized 
Table 4. Best performed DFT functionals for the calculation of bond length, vibrational frequency and atomization energy of metal dimers

\begin{tabular}{cccc}
\hline Metal dimers & Bond length & $\begin{array}{c}\text { Vibrational } \\
\text { frequency }\end{array}$ & $\begin{array}{c}\text { Atomization } \\
\text { energy }\end{array}$ \\
\hline $\mathrm{Al}_{2}$ & BHandH & M05-2X & BLYP, B3LYP \\
$\mathrm{Ti}_{2}$ & M06-L & M06-L & M06-2X \\
$\mathrm{V}_{2}$ & TPSS & TPSS & M05-2X \\
$\mathrm{Cr}_{2}$ & BLYP & BLYP & M06-2X \\
$\mathrm{Mn}_{2}$ & B3P86 & B3P86 & MN12-L \\
$\mathrm{Fe}_{2}$ & BPW91, B97D & N12 & MN12-SX \\
$\mathrm{Co}_{2}$ & MN12-SX & MN12-L & BHandH \\
$\mathrm{Ni}_{2}$ & M05 & M06-2X & BHandH \\
$\mathrm{Cu}_{2}$ & M06-2X & M06-2X & B3LYP \\
$\mathrm{Zn}_{2}$ & MN12-L & BHandH & M06-2X \\
\hline
\end{tabular}

empirical exchange correlation functional M06-2X coincides very well with the experimental results. ${ }^{[65]}$ However, M06-2X functional predicts higher $\mathrm{Ni}-\mathrm{Ni}$ bond lengthin $\mathrm{Ni}_{2}$ dimer. This deviation could be minimized using a higher basis set with M06-2X functional. In summary, Minnesota functional shows good performance for both bond length and vibrational frequency of $\mathrm{Ni}_{2}$ dimer. In the case of atomization energy, the hybrid $\mathrm{BHandH}$ functional predicts energy closer to experimental results. ${ }^{[66]}$ All the other studied functionals overestimate the atomization energy.

\section{$\mathrm{Cu}_{2}$ Dimer}

Present calculations have shown that, Minnesota functional M06-2X gives closer results than other studied functionals for $\mathrm{Cu}-\mathrm{Cu}$ bond length in $\mathrm{Cu}_{2}$ dimer. The difference between experimental[67] and theoretical values is small. Earlier Pou-Amerigo et al., ${ }^{[68]}$ have performed the CASPT2 studies on $\mathrm{Cu}_{2}$ dimer, and have obtained accurate bond length, vibrational frequency and atomization energy. From my studies, it is observed that all the studied functionals overestimate the vibrational frequency. It must be noted that Gutsev and Bauschlicher have predicted better vibrational frequency values for $\mathrm{Cu}_{2}$ dimer. This indicates that, using higher level basis set one may get the better vibrational frequency in this dimer. In the case of atomization energy, all the GGA functionals give higher energy. The hybrid version of GGA functionals gives consistent result in comparison with experimental value. For instance, the atomization energy, calculated by hybrid B3LYP functional gives $2.24 \mathrm{eV}$, which coincides well with the experimental value of $2.01 \pm 0.08 \mathrm{eV} .{ }^{[7]}$ This indicates that, the incorporation of Hartree-Fock exchange part in the exchange correlation potential could yield better results in atomization energy of $\mathrm{Cu}_{2}$ dimer. Furthermore, in accordance with Pou-
Amerigo et al., it is necessary to use higher basis set or highly correlated ab initio method to get accurate structural and energy parameters in the $\mathrm{Cu}_{2}$ dimer.

\section{$\mathrm{Zn}_{2}$ dimer}

A local functional with the contribution of $0 \% \mathrm{HF}$ exchange MN12-L yields better result for $Z n-Z n$ bond length of $Z n_{2}$ dimer than the other DFT functionals that are taken for my study. When compared with experimental results[ ${ }^{[69]}$ all other DFT functionals considered in this study overestimate $\mathrm{Zn}-\mathrm{Zn}$ bond length. The calculated results are consistent with Gutsev and Bauschlicher study. ${ }^{[20]}$ They found that GGA functionals overestimate $\mathrm{Zn}-\mathrm{Zn}$ bond length. In the case of vibrational frequency, the frequency calculated by hybrid $\mathrm{BHandH}$ functional coincides very well with the experimental result. The experimental vibrational frequency has been obtained by Givan and Loewenschuss. ${ }^{[70]}$ Further, Su et al. have calculated the atomization energy experimentally. ${ }^{[71]}$ The atomization energy calculated by the studied DFT functionals gives consistent result in this study. In particular, Minnesota functionals M05-2X and M06-2X functionals gives closer result in comparison with the experimental data. However, the difference between experimental and theoretical value is $\sim 0.05 \mathrm{eV}$. This difference could be minimized using higher basis set. In summary, based on the overall results Minnesota functionals could be recommended for the structure and energy studies of $\mathrm{Zn}_{2}$ dimer.

\section{General Performance of DFT Functionals}

To visualize the generalized performance of DFT functionals, the MAD error for all studied metal dimers have been calculated. The MAD error of bond length, vibrational frequency and atomization energy are shown in Figure 1 . The selected DFT functionals are given in the X-axis, and the MAD error of bond length, vibrational frequency and atomization energy are given in $\mathrm{Y}$-axis. For the clear understanding of difference in the performance between the functionals, the $Y$-axis initial value is taken as $0.10 \AA$ and $125 \mathrm{~cm}^{-1}$ for bond length and vibrational frequency respectively. The DFT functionals BLYP, TPSS, MN12-L, M05, M06, M06-L, TPSSh, MN12-SX give less deviation ( $<0.15 \AA$ ) in the case of bond length. The functionals, BHandH, M05-2X and M06-2X give large deviation ( $>0.20 \AA$ ). The Minnesota functionals produce mixed results. Some functionals, such as MN12-L, M05, M06, M06-L, and MN12-SX produce better results and some functionals namely M05-2X and M06-2X produce large MAD error. From the above results, it could be noted that, Minnesota functionals with less percentage of HF exchange shows overall performance to be better in the case of Metal-Metal bond length. On the other hand, functionals M05-2X and M06-2X show overall poor performance, these functionals include a high percentage of $\mathrm{HF}$ 
exchange. When considering vibrational frequency, all thefunctional staken for this study showlarger deviation (150 $\mathrm{cm}^{-1}$ ) from their corresponding experimental values. Among them, less deviation has been noted for M06-L and M05 functionals. In the case of atomization energy, it is noted that GGA functionals producea large deviation. The functionals MN12-SX and $\mathrm{BHandH}$ give better results, i.e. smaller deviation.

Table 4 summarizes the best performed functionals for the calculation of bond length, vibrational frequency and atomization energy of the studied metal dimers. The best performing functional among different functionals that are taken in this study is one, which produces very less deviation from their corresponding experimental values. From Table 4, it can be noted that, no specific functional showsgood performance for all the studied parameters such as bond length, vibrational frequency and atomization energy. Similarly, no functional shows good performance in studying all the metal dimers. However, some Minnesota functionals show good performance in bond length and vibrational frequency calculations. In summary, Minnesota functionals show decent performance for all the studied metal systems.

\section{CONCLUSIONS}

The difference in the performance of DFT functionals ranging from GGA to hybrid meta-GGA along with Minnesota functionals have been assessed for the studies on bond length, vibrational frequency, and atomization energy of some catalytically important metal dimers. It is noted that mixed results were produced by these functionals used in this study. The accuracy of the calculated results varies for each metal dimer, which highly depends upon the selection of functional and basis set. My calculations have shown that, the basis set plays an important role in the study of metal systems. One has to use higher basis set or highly correlated $a b$ initio method to get accurate theoretical results in some metal dimers. On the other hand, the lower level basis set will be enough to calculate the structural and energetical properties of some metal dimers. The Metal-Metal bond length, in the dimers $\mathrm{Al}_{2}, \mathrm{Mn}_{2}, \mathrm{Fe}_{2}$, and $\mathrm{Ni}_{2}$ can be studied using lower level basis set. In these dimers, selection of functional is important for predicting the structure. Furthermore, one has to use a higher basis set to get the accurate geometrical parameters of all the other dimers. In the case of vibrational frequency calculation, the functionals with 6-31G(d) basis set predict better results for $\mathrm{Al}_{2}, \mathrm{Mn}_{2}$ and $\mathrm{Zn}_{2}$ dimer. But for the other dimers that are taken in my study, all the studied functionals overestimate the vibrational frequency values, when compared with their experimental data. In the case of atomization energy calculation, the functionals that are taken for my study predict accurate results for $\mathrm{Al}_{2}$, and $\mathrm{Co}_{2}$ dimers only. For the rest of the dimers, higher basis set or highly correlated $a b$ initio methods would be of better choice. As it is seen from the earlier studies, DFT functionals fail to calculate atom energy accurately and hence it results in higher atomization energy values. When considering the overall performance, Minnesota functionals yield good results for bond length, vibrational frequency and atomization energy for all the studied metal dimers. For instance, M06-L can be a good choice for the studies on bond length and vibrational frequency of selected metal dimers. MN12-SX, a screened-exchange (SX) hybrid functional with $25 \% \mathrm{HF}$ exchange in the short-range and $0 \%$ in the long-range can be recommended for studying atomization energy.

This study provides a generalized overview of recently developed DFT functionals and their performance with a lower level basis set. I believe that, this study could be of great helpto the experimentalists and theoreticians to perform future studies on these metal systems.

Acknowledgment. The author is thankful to the management of Kalasalingam University for providing the financial support to establish the computational research facility at the international research centre (IRC), Kalasalingam University.

\section{REFERENCES}

[1] J. Trost, H. Brune, J. Wintterlin, R. J. Behm, G. Ertl, J. Chem. Phys. 1998, 108, 1740.

[2] R. Burgert, H. Schnockel, A. Grubisic, X. Li, S. T. Stokes, K. H. Bowen, G. F. Gantefor, B. Kiran, P. Jena, Science 2008, 319, 438.

[3] A. O. Orlov, I. Amlani, G. H. Bernstein, C. S. Lent, G. L. Snider, Science 1997, 277, 928.

[4] S. Keav, S. K. Matam, D. Ferri, A. Weidenkaff, Catalysts 2014, 4, 226.

[5] C. Ko, K. Kerman, S. Ramanathan, J. Power Sources 2012, 13, 343.

[6] A. Primo, T. Marino, A. Corma, R. Molinari, H. García, J. Am. Chem. Soc. 2011, 133, 6930.

[7] M. D. Morse, Chem. Rev. 1986, 86, 1049.

[8] J. R. Lombardi, B. Davis, Chem. Rev. 2002, 102, 2431.

[9] D. R. Salahub, Adv. Chem. Physics, Vol. 67: AB INITIO Methods Quantum Chem. II 2009, 142, 447.

[10] A. Kalemos, I. G. Kaplan, A. Mavridis, J. Chem. Phys. 2010, 132, 024309.

[11] A. Kalemos, A. Mavridis, J. Chem. Phys. 2011, 135, 134302.

[12] S. Yamamoto, H. Tatewaki, H. Moriyama, H. Nakano, J. Chem. Phys. 2006, 124, 124302. 
[14] E. M. Spain, M. D. Morse, J. Phys. Chem. 1992, 96, 2479.

[15] J. C. Pinegar, J. D. Langenberg, C. A. Arrington, E. M. Spain, M. D. Morse, J. Chem. Phys. 1995, 102, 666.

[16] S. Datta, M. Kabir, S. Ganguly, B. Sanyal, T. SahaDasgupta, A. Mookerjee, Phys. Rev. B. 2007, 76, 014429.

[17] C. J. Barden, J. C. Rienstra-Kiracofe, H.F. Schaefer, J. Chem. Phys. 2000, 113, 690.

[18] M. Brynda, L. Gagliardi, B. O. Roos, Chem. Phys. Lett. 2009, 471, 1.

[19] J. Du, X. Sun, H. Wang, Int. J. Quantum Chem. 2008, 108, 1505.

[20] S. Yanagisawa, T. Tsuneda, K. Hirao, J. Chem. Phys. 2000, 112, 545.

[21] G. L. Gutsev, C. W. Bauschlicher, J. Phys. Chem. 2003, 107, 4755.

[22] S. Paranthaman, K. Hong, J. Kim, D. E. Kim, T. K. Kim, J. Phys. Chem. A 2013, 117, 9293.

[23] A. D. Becke, Phys. Rev. A 1988, 38, 3098.

[24] C. Lee, W. Yang, R. G. Parr, Phys. Rev. B. 1988, 37, 785.

[25] J. P. Perdew, Phys. Rev. B. 1986, 33, 8822.

[26] J. P. Perdew, Electronic Structure of Solids, 1991, Akademic Verlag, Berlin, Germany.

[27] S. Grimme, J. Comput. Chem. 2006, 27, 1787.

[28] J. P. Perdew, K. Burke, M. Ernzerhof, Phys. Rev. Lett. 1996, 77, 3865.

[29] J. P. Perdew, M. Emzerhof, K. Burke, J. Chem. Phys. 1996, 105, 9982.

[30] C. Adamo, V. Barone, J. Chem. Phys. 1998, 108, 664.

[31] J. Tao, J. P. Perdew, V. N. Staroverov, G. E. Scuseria, Phys. Rev. Lett. 2003, 91, 146401.

[32] R. Peverati, D. G. Truhlar, J. Chem. Theory Comput. 2012, 8, 2310.

[33] Y. Zhao, D. G. Truhlar, J. Chem. Phys. 2006, 125, 194101.

[34] R. Peverati, D. G. Truhlar, Phys. Chem. Chem. Phys. 2012, 14, 13171.

[35] A. D. Becke, J. Chem. Phys. 1993, 98, 5648.

[36] J. P. Perdew, Y. Wang, Phys. Rev. B. 1992, 45, 13244.

[37] C. Adamo, V. Barone, J. Chem. Phys. 1999, 110, 6158.

[38] Y. Zhao, N. E. Schultz, D. G. Truhlar, J. Chem. Phys. 2005, 123, 161103.

[39] Y. Zhao, N. E. Schultz, D. G. Truhlar, J. Chem. Theory Comput. 2006, 2, 364.

[40] Y.Zhao, D. G. Truhlar, Theor. Chem. Acc. 2008, 120, 215.

[41] V. N. Staroverov, G. E. Scuseria, J. M. Tao, J. P. Perdew, J. Chem. Phys. 2003, 119, 12129.

[42] R. Peverati, D. G. Truhlar, Phys. Chem. Chem. Phys. 2012, 14, 16187.

[43] Gaussian 09, Revision E.01, M. J. Frisch, G. W. Trucks, H. B. Schlegel, G. E. Scuseria, M. A. Robb, J. R. Cheeseman, G. Scalmani, V. Barone, B. Mennucci, G. A. Petersson, H. Nakatsuji, M. Caricato, X. Li, H. P.
Hratchian, A. F. Izmaylov, J. Bloino, G. Zheng, J. L. Sonnenberg, M. Hada, M. Ehara, K. Toyota, R. Fukuda, J. Hasegawa, M. Ishida, T. Nakajima, Y. Honda, O. Kitao, H. Nakai, T. Vreven, J. A. Montgomery, Jr., J. E. Peralta, F. Ogliaro, M. Bearpark, J. J. Heyd, E. Brothers, K. N. Kudin, V. N. Staroverov, R. Kobayashi, J. Normand, K. Raghavachari, A. Rendell, J. C. Burant, S. S. Iyengar, J. Tomasi, M. Cossi, N. Rega, J. M. Millam, M. Klene, J. E. Knox, J. B. Cross, V. Bakken, C. Adamo, J. Jaramillo, R. Gomperts, R. E. Stratmann, O. Yazyev, A. J. Austin, R. Cammi, C. Pomelli, J. W. Ochterski, R. L. Martin, K. Morokuma, V. G. Zakrzewski, G. A. Voth, P. Salvador, J. J. Dannenberg, S. Dapprich, A. D. Daniels, Ö. Farkas, J. B. Foresman, J. V. Ortiz, J. Cioslowski, and D. J. Fox, Gaussian, Inc., Wallingford CT, 2009.

[44] M. B. Abreu, C. Powell, A. C. Reber, S. N. Khanna, J. Am. Chem. Soc. 2012, 134, 20507.

[45] M. F. Cai, T. P. Dzugan, V. E. Bondybey, Chem. Phys. Lett. 1989, 155, 430.

[46] N. Drebov, R. Ahlrichs, J. Chem. Phys. 2011, 134, 124308.

[47] M. Doverstal, B. Lindgren, U. Sassenberg, C. A. Arrington, M. D. Morse, J. Chem. Phys. 1992, 97, 7087.

[48] O. Hübner, H.-J. Himmel, L. Manceron, W. Klopper, J. Chem. Phys. 2004, 121, 7195.

[49] C. Cosse, M. Fouassier, T. Mejean, M. Tranquille, D. P. DiLella, M. Moskovits, J. Chem. Phys. 1980, 73, 6076.

[50] L. M. Russon, S. A. Heidecke, M. K. Birke, J. Conceicao, M. D. Morse, P.B. Armentrout, J. Chem. Phys. 1994, 100, 4747.

[51] E. M. Spain, J. M. Behm, M. D. Morse, J. Chem. Phys. 1992, 96, 2511.

[52] S. P. Walch, C. W. Bauschlicher, B. O. Roos, C. J. Nelin, Chem. Phys. Lett. 1983, 103, 175.

[53] V. E. Bondybey, J. H. English, Chem. Phys. Lett.94 (1983) 443.

[54] S. M. Casey, D. G. Leopold, J. Phys. Chem. 1993, 97, 816.

[55] K. Hilpert, R. Ruthardt, Berichte Der Bunsenges. Für Phys. Chem. 1987, 91, 724.

[56] G. Mills, M. S. Gordon, H. Metiu, Chem. Phys. Lett. 2002, 359, 493.

[57] S. A. Varganov, R. M. Olson, M. S. Gordon, G. Mills, H. Metiu, Chem. Phys. Lett. 2003, 368, 778.

[58] M. Cheeseman, R. J. V. Zee, H. L. Flanagan, W. Weltner, J. Chem. Phys. 1990, 92, 1553.

[59] A. D. Kirkwood, K. D. Bier, J. K. Thompson, T. L. Haslett, A. S. Huber, M. Moskovits, J. Phys. Chem. 1991, 95, 2644.

[60] H. Purdum, P. A. Montano, G. K. Shenoy, T. Morrison, Phys. Rev. B. 1982, 25, 4412. 
[61] M. Moskovits, D. P. DiLella, J. Chem. Phys. 1980, 73, 4917.

[62] S. K. Loh, L. Lian, D. A. Hales, P. B. Armentrout, J. Phys. Chem. 1988, 92, 4009.

[63] A. Kant, B. Strauss, J. Chem. Phys. 1964, 41, 3806.

[64] J.-G. Dong, Z. Hu, R. Craig, J. R. Lombardi, D. M. Lindsay, J. Chem. Phys. 1994, 101, 9280.

[65] I. Shim, K. A. Gingerich, J. Chem. Phys. 1984, 80, 5107.

[66] H. Wang, H. Haouari, R. Craig, J. R. Lombardi, D. M. Lindsay, J. Chem. Phys. 1996, 104, 3420.

[67] M. D. Morse, G. P. Hansen, P. R. R. Langridge-Smith, L.-S. Zheng, M. E. Geusic, D. L. Michalopoulos, R. E. Smalley, J. Chem. Phys. 1984, 80, 5400.
[68] R. S. Ram, C. N. Jarman, P. F. Bernath, J. Mol. Spectrosc. 1992, 156, 468.

[69] R. Pou-Amerigo, M. Merchan, I. Nebot-Gil, P.-A. Malmqvist, B. O. Roos, J. Chem. Phys. 1994, 101, 4893.

[70] K. D. Carlson, K. R. Kuschnir, J. Phys. Chem. 1964, 68, 1566.

[71] A. Givan, A. Loewenschuss, Chem. Phys. Lett. 1979, 62, 592.

[72] C.-H. Su, P.-K. Liao, Y. Huang, S.-S. Liou, R. F. Brebrick, J. Chem. Phys. 1984, 81, 11.

[73] Z. Fu, G. W. Lemire, G. A. Bishea, M. D. Morse, J. Chem. Phys. 1990, 93, 8420. 